\title{
Rentas Mínimas de Inserción de las Comunidades Autónomas: una visión conjunta de su evolución y alcance
}

\author{
Ana Arriba*
}

Resumen: En este artículo se lleva a cabo una aproximación evaluativa al conjunto de programas autonómicos de Rentas Mínimas de Inserción (RMI). Estos programas ofrecen una prestación de garantía mínima de ingresos en situaciones de necesidad probada destinada a la inserción social de sus perceptores. En el texto se estudia el particular proceso de implantación de los RMI por parte de los gobiernos autonómicos así como las trayectorias que han seguido con posterioridad. Estos procesos explican en buena medida los límites para evaluar su alcance. A pesar de lo cual, es posible, a partir de la evidencia disponible (indicadores de esfuerzo presupuestario, intensidad y cobertura), señalar que las reformas y transformaciones que han seguido las RMI en la última década no solo no han mejorado su escasa capacidad protectora frente a la pobreza sino que han incrementado su heterogeneidad y fragmentación. Las conclusiones indican que el refuerzo y la armonización de la acción protectora de los RMI, junto con un esfuerzo de coordinación de los diferentes agentes implicados, contribuirán a la mejora de la lucha contra la pobreza y la exclusión social en España.

Palabras clave: Rentas Mínimas de Inserción, asistencia social, inclusión social, activación, reformas de política social, evaluación de políticas sociales.

\section{INTRODUCCIÓN: LAS RENTAS MÍNIMAS DE INSERCIÓN}

Los programas de renta mínima o de garantía de ingresos mínimos son prestaciones económicas concebidas para que ningún ciudadano se quede sin nada para vivir. Tienen como rasgo común, aunque no único, ofrecer un ingreso mínimo garantizado de forma complementaria o subsidiaria a otros sistemas de protección social y

* $\quad$ Este trabajo se ha realizado gracias a la participación de la autora en los proyectos de investigación NURSOPOB ("Nuevos Riesgos Sociales y Trayectorias de las Políticas del Bienestar", Plan Nacional de I+D+I, MEC, SEJ2005-06599) e INMEBEA (“Inmigración y Estado de Bienestar en la España Autonómica”, Plan Nacional de I+D+i, SEJ2007-67521/CPOL). 
son prestaciones de asistencia social, es decir, para acceder a ellas se ha de demostrar la insuficiencia de recursos (means-tested). Además, suelen incluir otros requisitos relativos a residencia o a edad, destinados a limitar el acceso de determinados sectores de la población (extranjeros, jóvenes... ) y exigir ciertas condiciones como la participación en actividades de apoyo o de acceso al empleo (Euzeby, 1991). Su finalidad es cubrir un nivel básico mientras dure la situación de necesidad o pobreza por lo que constituyen una "última red" de protección social.

En los Estados de Bienestar europeos, el incremento de las cifras de desempleo en los años ochenta impulsó el protagonismo de estas políticas que han seguido desde entonces una filosofía de doble derecho -a la garantía de ingresos y a la inserción social- siguiendo el ejemplo del programa francés de Revenú Minimum d'Insertion. Desde esta perspectiva, se conciben como mecanismos de la lucha contra la exclusión social o instrumentos de recomposición del vínculo social, lo que se materializa en la introducción de mecanismos o incentivos para la participación social y laboral, en sintonía con la orientación hacia la activación, predominante en las políticas sociales europeas (Aust y Arriba, 2005; Arriba y Pérez-Eransus, 2007; Moreno y Serrano, 2007).

En España, este tipo de políticas ha sido impulsado por las Comunidades Autónomas que, a finales de los años ochenta y comienzos de los noventa, pusieron en marcha una serie de programas destinados a personas potencialmente activas y sus familias, conocidas como Rentas Mínimas de Inserción (RMI). En general, éstas ofrecen una protección de baja intensidad, condicionada al cumplimiento de una serie de requisitos y marcada por la discrecionalidad en su aplicación. Siguen, con serias restricciones, la filosofía del doble derecho y constituyen el último mecanismo de la protección social española. Los programas autonómicos de RMI son uno de los mecanismos de un conjunto de prestaciones asistenciales dispersas en los diversos sistemas de protección -pensiones, desempleo o servicios sociales-y niveles de la administración pública. Las prestaciones asistenciales españolas ofrecen en conjunto una protección de baja intensidad protectora jerarquizada según la relación pasada y presente con el mercado de trabajo (Arriba y Guinea, 2008).

En este artículo se lleva a cabo una aproximación evaluativa al alcance conjunto de los programas autonómicos de RMI. Se toma como punto de partida su peculiar proceso de implantación y su heterogénea trayectoria durante la última década para comprender las dificultades prácticas para llevar a cabo una evaluación conjunta de estos programas. El texto prosigue con la valoración conjunta del alcance de estos programas y del efecto de sus últimos cambios normativos sobre su capacidad protectora y activadora.

\section{POLÍTICAS AUTONÓMICAS DE RENTAS MÍNIMAS DE INSERCIÓN}

La política autonómica de RMI tiene su origen a finales de los años ochenta en un 82 contexto de expansión de las políticas de asistencia social en España. En aquel mo- 
mento las Comunidades Autónomas elaboraron e implementaron una serie de programas de garantía mínima de ingresos dirigidos a la lucha contra la pobreza y la exclusión social. Las Comunidades Autónomas habían generado ya un espacio propio de acción frente a la pobreza a través de sus Estatutos de Autonomía y de sus Leyes de Servicios Sociales. La Constitución Española de 1978 había establecido la asistencia social como una competencia de las 17 Comunidades Autónomas (art. 148), mientras reservó la responsabilidad sobre legislación y el régimen económico de la Seguridad Social al Gobierno Central. Eso sí, la Constitución no definió cuáles eran los contenidos de la asistencia social, lo que ha permitido a las Comunidades Autónomas incluir en sus Estatutos de Autonomía un gran número de servicios y funciones de asistencia social, servicios sociales, bienestar social, desarrollo comunitario y promoción social.

Entre 1989-1993, el proceso de implantación de estos programas tuvo lugar de forma concatenada entre las Comunidades Autónomas, una vez que el Gobierno vasco anunció su Plan de Lucha contra la Pobreza en 1988. Hasta la aprobación del programa en Aragón en junio de 1993, el proceso tuvo un fuerte carácter mimético y competitivo en el que las Comunidades Autónomas emplearon argumentos e ideas europeístas (recomendaciones de las instituciones europeas o la adopción de políticas similares por otros Estados de Bienestar) para legitimar su actuación frente a las críticas del Gobierno central. En esta dinámica, los sindicatos, algunas organizaciones no gubernamentales y en algunos casos, los expertos académicos y profesionales del área fueron especialmente activos en la difusión de la medida y en la presión sobre los gobiernos autónomos (Arriba, 2002).

A mediados de la década de los noventa, una serie de investigaciones mostró que, dada la heterogeneidad de estos programas, hablar de la política de RMI española era forzar la realidad (Aguilar, Gaviria, y Laparra, 1995; Ayala, 2000; Estévez, 1998). El nexo común de todos ellos era ofrecer un ingreso mínimo garantizado para individuos entre los 25 y los 65 años, al que se accede mediante demostración de recursos insuficientes de la unidad de convivencia (means-tested) y para el cual se requiere la residencia legal de los solicitantes (u otros mecanismos orientados a restringir la movilidad entre Comunidades Autónomas). Eran prestaciones de cuantía diferencial (de la cuantía a percibir se restan los ingresos ya existentes en el hogar), por un periodo de tiempo determinado con diferentes posibilidades de prórroga. Se financiaban por las propias Comunidades Autónomas y gestionaban en el marco de sus sistemas de servicios sociales, principalmente la atención primaria, bajo la responsabilidad del nivel local de la Administración. También compartían una orientación programática hacia la inserción social de sus beneficiarios, al estilo del programa francés de RMI.

Estas investigaciones mostraron la diversidad de normativas reguladoras (leyes, decretos, órdenes), de niveles de intensidad y cobertura, de duraciones de la prestación y disponibilidad presupuestaria que daban lugar a distintos niveles de garantía del derecho social. Únicamente el grado de desarrollo del programa del País Vasco permitía considerarlo RMI en sentido pleno, es decir como una prestación 
de derecho no limitada por discrecionalidad técnica o política ni por disponibilidades financieras. Un grupo de programas contenía elementos restrictivos en su acceso y uso por lo que solo podría calificarse RMI en sentido limitado. Un tercer grupo tenía fuertes restricciones en su carácter de derecho por lo que no cabía considerarlo como RMI. Esta diversidad también se materializaba en las formas en las que se planteaba el vínculo entre los objetivos de protección frente a la pobreza y de inserción social, que distinguía entre programas de RMI, de empleo social protegido y de prestaciones de asistencia social. Otros estudios se centraron en la prácticas de aplicación de estos programas autonómicos, mostrando cómo los encargados de su puesta en marcha reinterpretan y adaptan los objetivos de los programas, flexibilizan o endurecen los requisitos y condiciones en función de las diferentes situaciones de los solicitantes, con un grado de discrecionalidad necesario para su funcionamiento en un contexto de indefinición programática y fuerte restricción presupuestaria (Serrano y Arriba, 1998).

A mediados de los noventa el periodo de expansión de la asistencia social y del gasto social dio paso a otro caracterizado por la preocupación por la racionalización, el mantenimiento y la viabilidad del sistema de protección social, en especial en el aseguramiento de su financiación (Rodríguez-Cabrero, 2004). En este nuevo contexto, las RMI autonómicas han seguido un itinerario desigual de reformas, calificado como "travesía del desierto” (Laparra, 2004). Desde 1995 algunas Comunidades Autónomas han llevado a cabo importantes reformas, reestructuraciones e incluso refundaciones de sus programas de RMI, al tiempo que otras los han mantenido prácticamente inalterados. La evolución posterior a 1995 (ver Cuadro 1) muestra que la actividad de reforma o reestructuración de los programas RMI ha sido constante pero, a diferencia del periodo de puesta en marcha no tiene carácter unitario ni concatenado como denotan su desigual aparición en el calendario y la diversidad de sus contenidos.

A pesar de lo cual, se pueden observar algunas pautas comunes entre las diversas reformas de RMI de la última década. Entre ellas, su inserción en marcos programáticos, como los Planes transversales o multisectoriales para la inclusión social que se refieren a amplios sectores de la política autonómica. En la década 1998-2007 al menos trece Comunidades Autónomas han contado con Planes de este tipo y en todos ellos se contempla algún tipo de reelaboración de las RMI, desde el aumento de sus cuantías y coberturas hasta su consolidación normativa pasando por reformas en las condiciones y requisitos de acceso. En esta trayectoria, es inevitable considerar la influencia de la dinámica europea: en junio del año 2000 la Comisión y el Consejo Europeo lanzaron la Estrategia de Lisboa para la inclusión social y, en ella, los estados miembro se comprometían a elaborar Planes Nacionales para la Inclusión Social (PNAInc) con periodicidad bianual. Los primeros PNAInc españoles señalaron que los Planes Territoriales para la Inclusión, a desarrollar por las Comunidades Autónomas, eran un instrumento clave en el compromiso español con la inclusión social. En el año 2000 muchos de estos Planes Territoriales o autonómicos estaban ya en marcha (como País Vasco, Islas Canarias, Castilla y León, Castilla-La Mancha, Cataluña, La Rioja y Navarra) o esta84 ban siendo elaborados (Madrid) por lo que la iniciativa europea reforzó esta tenden- 
cia. Eso sí, los Planes elaborados con posterioridad recogen la terminología, estructura y objetivos de la Estrategia Europea (Pérez-Eransus, Arriba y Parrilla, 2009).

La mejora de las normativas reguladoras de muchas de las RMI constituye otra de las pautas comunes del último periodo. Las RMI han sido reconocidas como derecho en las reformas de los Estatutos de Autonomía de seis Comunidades Autónomas (Andalucía, Aragón, Baleares, Castilla y León, Cataluña, y Comunidad Valenciana). Aún más importante y significativa ha sido su regulación a través de una ley de muchas de las RMI autonómicas, como es el caso de Asturias, Cataluña, Galicia, Madrid o Navarra y en 2007 Canarias, Cantabria y Murcia. En este aspecto, la política del País Vasco, con cuatro leyes reguladoras en este periodo, mantiene su trayectoria de progreso y continuo desarrollo. Desde mediados de los años noventa, los expertos e interesados en estas políticas han interpretado que la regulación a través de ley de las prestaciones RMI supone el reconocimiento de un derecho, un ascenso en la jerarquía protectora de las diferentes RMI.

Más difícil resulta extraer conclusiones válidas sobre si estas reformas han supuesto una ampliación de los requisitos y/o una flexibilización de las condiciones de estos programas a mediados de la década de los noventa. En algunos casos, se han redefinido requisitos importantes para la percepción de la prestación como la edad (País Vasco), la unidad de convivencia (Madrid o Navarra) o se han distinguido itinerarios según el nivel de empleabilidad (Cataluña). Sin embargo, en otras reformas solo se han realizado pequeños ajustes en las condiciones de acceso y disfrute de las prestaciones (indexación de cuantías, establecimiento de máximos y mínimos o ampliación de los límites de duración de la prestación).

Sí es posible observar una tendencia general, convergente con la Estrategia Europea, como es la del uso del término exclusión social no solo referido a la concepción del problema (objeto de la intervención) sino también a las estrategias de acción, orientadas a la inclusión social. Por una parte, esto conlleva la puesta en marcha de programas más allá de la garantía de ingresos en sectores de políticas de empleo, vivienda, salud o educación, a través de los ya referidos Planes transversales.

Por otra, significa la coherencia de estas reformas con una de las tendencias de transformación de la política social europea (Taylor-Gooby, 2004): la tendencia a la activación, en especial de la población capaz de acceder a un empleo. A pesar de que la influencia en las RMI españolas de la tendencia activadora es creciente, se encuentra aún más en un plano discursivo que real, con un gasto considerablemente menor que el dedicado a las prestaciones (Arriba, 2004). La forma de concebirlo y llevarlo a la práctica es divergente: desde la filosofía del doble derecho (País Vasco, Madrid o Navarra) hasta la idea de la contraprestación en pago de la deuda que se contrae con la sociedad. Los mecanismos empleados han estado sobre todo destinados a favorecer la inserción laboral de los perceptores RMI a través servicios de formación y orientación para el empleo, programas de empleo social protegido, empresas de inserción o incentivos al empleo. La limitada intervención pública se ha basado en buena medida en la actuación de entidades de iniciativa social (Pérez-Eransus, 2005). 


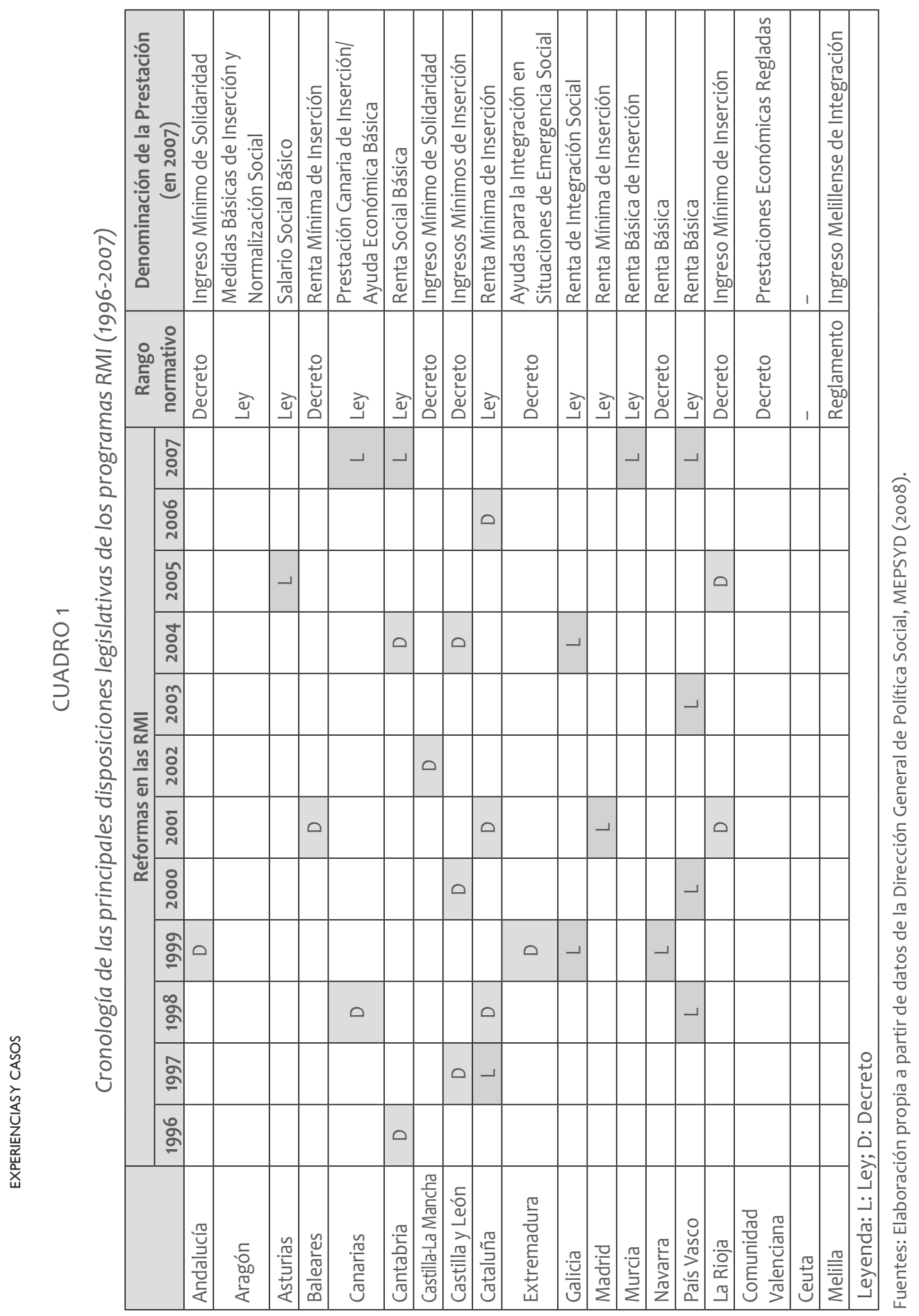




\section{LOS LÍMITES PARA UNA EVALUACIÓN CONJUNTA DE LAS RMI}

Antes de valorar el alcance de las RMI y de sus últimas reformas, se va a considerar brevemente cómo las características de estos programas junto con su particular desarrollo han dificultado y dificultan la práctica evaluadora. Me centraré en especial en dos tipos de problemas: las cuestiones relativas a la escasa definición de su objeto de intervención y las relacionadas con el liderazgo de las Comunidades Autónomas en su puesta en marcha.

En relación con el primero, el objetivo de las rentas mínimas se ha centrado en la lucha contra la exclusión social dejando en segundo plano su papel como mecanismo de protección en situaciones de pobreza. El concepto de exclusión social carece de una definición compartida y resulta de difícil operacionalización, lo que dificulta la medición de los resultados e impactos de estas políticas.

Así, la concepción del problema de la exclusión social se aborda desde perspectivas que comparten una perspectiva multidimensional pero que definen de forma divergente el ámbito y alcance de la exclusión social. Por un lado, hay autores que definen y estudian la exclusión social como un riesgo que afecta a amplios sectores de la población y que tiene que ver no solo con desigualdades económicas, sino también con desventajas en distintos ámbitos -empleo, ingresos, educación, vivienda o la participación social (EAPN, 2006). Otros identifican la exclusión social con un colectivo de menor tamaño, que afecta a aquellas personas que acumulan junto con la ausencia de ingresos otros problemas graves en ámbitos como el empleo, las relaciones sociales, el ámbito educativo, la vivienda o la salud (VV.AA., 2008; Laparra et al., 2007; Pérez-Yruela et al., 2004). A este problema inicial hay que añadir la falta de indicadores referidos a los objetivos de inclusión, de activación y, sobre todo, al carácter dinámico del fenómeno. Esta divergencia en las formas de comprender el problema se refleja también entre los actores que elaboran, gestionan y evalúan los propios programas de RMI (Serrano y Arriba, 1998).

En último extremo hay cierto grado de acuerdo en su carácter multidimensional pero la falta de consenso en la definición y en sus indicadores determina con frecuencia la utilización indistinta de los términos pobreza y exclusión y el recurso, a modo de aproximación, a indicadores vinculados a la pobreza.

El segundo tipo de problemas se refieren a la forma en que se han puesto en marcha en España: su implantación a nivel autonómico bajo el liderazgo de las Comunidades Autónomas se materializa en información escasa y poco homogénea. Las prácticas de las Comunidades Autónomas de recopilación y diseminación de información sobre sus RMI son heterogéneas y, con frecuencia están orientadas a legitimar su actuación ante otras partes interesadas o ante sus propias opiniones públicas. 
Desde el año 2000, la Dirección General de Política Social², DGPS-MEPSYD, recopila anualmente información básica relativa a las prestaciones de las RMI autonómicas como parte de las actividades de los PNAInc. Da continuidad a un trabajo recopilador que puntualmente habían ofrecido otros autores (Ayala, 2000; Aguilar, Gaviria y Laparra, 1995). El informe se elabora a partir de datos agregados, no armonizados, por lo que se recoge información construida con definiciones no homogéneas. Esta recopilación se refiere únicamente a los aspectos relativos a las prestaciones de garantía de mínimos y no recoge datos relativos a las formas y presupuestos dedicados a las actividades orientadas a la inclusión social, una tarea aún más complicada.

En cuanto a las prácticas evaluativas de las Comunidades Autónomas, estas son discontinuas y, salvo excepciones, orientadas a la gestión administrativa. Solo excepcionalmente, las Comunidades Autónomas Ilevan a cabo evaluaciones globales (por lo general en momentos de reforma o cambio como en Navarra, Asturias o Madrid) y estudios concretos sobre sus perceptores o sobre algunos aspectos del funcionamiento del programa. No resulta fácil acumular conocimiento a partir de este tipo de trabajos, elaborado con objetivos y diseños diversos además de muy discontinuos en el tiempo. En términos generales, existe más información sobre aquellas RMI que tienen mayor grado de desarrollo, normativo y presupuestario.

De esta forma, los trabajos realizados desde el ámbito académico3, a los que se ha hecho referencia en el punto anterior (Aguilar, Gaviria, y Laparra, 1995; Ayala, 2000; Estévez, 1998), se han convertido en la mejor fuente de información sobre estos programas. Muchos de los cuales, ante la dificultad para recopilar información detallada, emplean una estrategia basada en el estudio de casos especialmente significativos o críticos o simplemente accesibles (Serrano y Arriba, 1998; Arriba, 2002; Ayala y Rodríguez, 2007; Pérez-Eransus, 2005). Todos estos trabajos se encuentran con la dificultad de llegar a conclusiones generalizables al conjunto de las RMI, dada la divergencia de itinerarios que estas han seguido.

Por último y, como se verá a continuación, el pobre desarrollo en muchas de las RMI hace que la tarea evaluativa carezca de sentido: en una buena parte de las Comunidades Autónomas, la RMI existe principalmente sobre el papel con un desarrollo casi inexistente.

2 En la actualidad inserta en el Ministerio de Sanidad y Política Social, anteriormente en el Ministerio de Educación, Política Social y Deporte, MEPSYD y en la del Ministerio de Trabajo y Asuntos Sociales, bajo diferentes denominaciones.

3 Algunos estrechamente ligados a la propia gestión de los programas, al ser encargados y/o financiados por la propia Administración. En el caso de estas últimas, mantienen una perspectiva de estudio de necesidades sin estar orientadas a evaluar sus resultados o su impacto. Véase Pérez-Yruela et al. (2002) y Pérez-Yruela et al. (2004) en Asturias; Aguilar et al. (2002) en Madrid y Gobierno de Navarra (1999) en 88 Navarra. También Estudios de Pobreza del País Vasco <http://www.siis.net/epds/documentos-e-informes>. 


\section{ALCANCE DE LAS RMI TRAS EL ÚLTIMO PERIODO DE REFORMAS}

A continuación se evalúa cuál es el alcance que el conjunto de las RMI tiene como último mecanismo de protección social, empleando los datos recogidos por la DGPS-MEPSYD desde el año $2000^{4}$. La cuestión principal es saber si las reformas seguidas por las RMI desde mediados de los años noventa, en especial la mejora de su estatus legal (reconociendo derechos y eliminando discrecionalidad y limitaciones presupuestarias) han traído consigo cambios significativos en la garantía del derecho en términos cuantitativos. Para ello analizaremos la evolución de tres indicadores relativos al gasto de las Comunidades Autónomas en estas prestaciones, a su cobertura y su intensidad protectora en el periodo 1997-2007.

En cuanto al primero, la evolución del gasto en términos absolutos (Gráfico 1) que las Comunidades Autónomas han dedicado a esta política ha sido incremental: el gasto total ha pasado de superar ligeramente los 160 millones de euros anuales en 1997 a alcanzar casi los 417 millones de euros en 2007, en un periodo caracterizado por la bonanza socioeconómica del país. La cobertura global, por otra parte, también ha ascendido, aunque de forma más moderada e irregular, pasando de 63.714 a 103.071 beneficiarios (hogares perceptores) en el mismo periodo.

\section{GRAFICO 1}

Evolución del gasto y cobertura totales en RMI

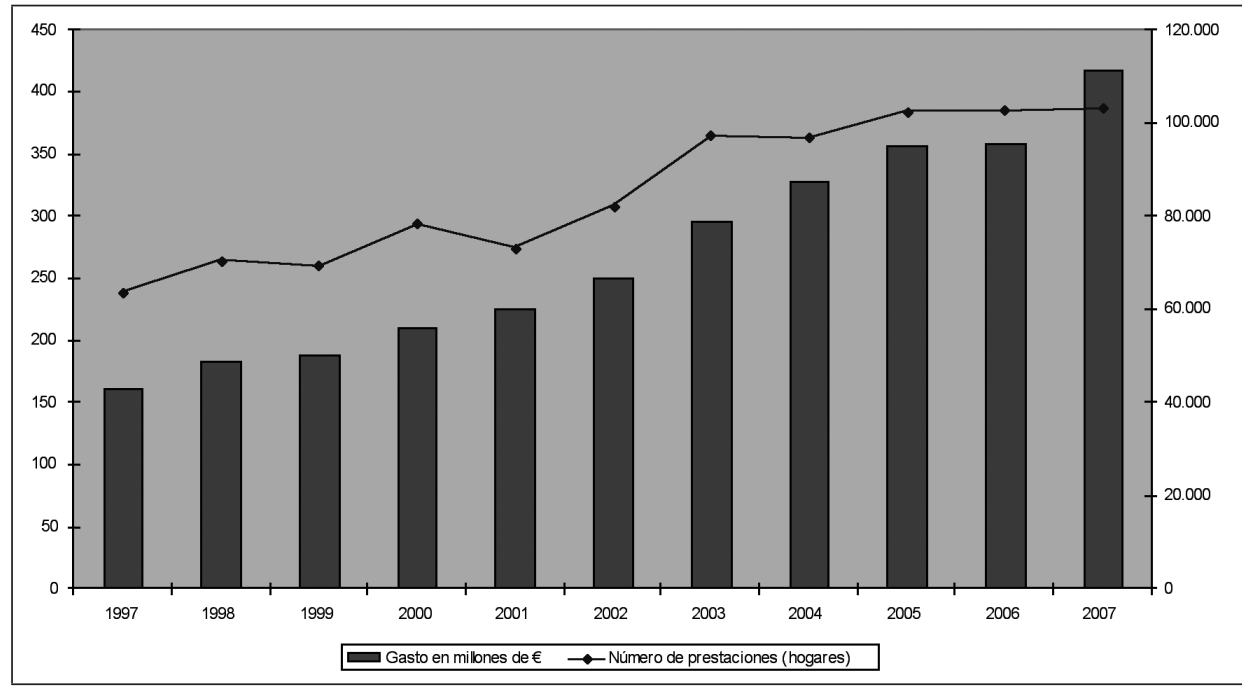

Fuentes: Elaboración propia a partir de datos de la Dirección General de Política Social, MEPSYD (2008).

4 Para años anteriores se recurre a los datos de Ayala (2000). 
Al examinar las cifras de gasto por Comunidades Autónomas en 2007, se observa una importante desigualdad y, por lo tanto, la desigual prioridad que los ejecutivos autonómicos otorgan a sus RMI (Cuadro 2). Así, para un grupo de Comunidades Autónomas, el gasto es prácticamente simbólico ya que no supera el 0,05\% de los presupuestos autonómicos (Aragón, Canarias, Cantabria, Castilla-La Mancha, Extremadura, Murcia, La Rioja o Valencia). Los niveles más elevados de gasto alcanzan entre el 0,2 y 0,5\% (Asturias, Cataluña, Madrid o Melilla). Finalmente, la excepción la constituye el programa vasco al que se dedica un $2,13 \%$ del presupuesto autonómico, lo que indica que nos encontramos ante un programa de una naturaleza diferente. En este sentido, cabe observar que el gasto total en RMI se debe a la contribución de un grupo de Comunidades Autónomas: por ejemplo, el presupuesto vasco para RMI supone prácticamente el $45 \%$ del gasto autonómico en estos programas.

\section{CUADRO 2}

Esfuerzo presupuestario y cobertura protectora de las prestaciones de RMI autonómicas 2007

\begin{tabular}{|c|c|c|c|c|}
\hline & $\begin{array}{c}\text { Gasto ejecutado } \\
\text { (millones de euros)* }\end{array}$ & $\begin{array}{c}\% \\
\text { sobre el } \\
\text { gasto** }\end{array}$ & $\begin{array}{c}\mathrm{N} .^{\circ} \text { de } \\
\text { beneficiarios }\end{array}$ & $\begin{array}{c}\% \\
\text { sobre } \\
\text { hogares*** }\end{array}$ \\
\hline Andalucía & 37,7 & $0,13 \%$ & 18.838 & $0,69 \%$ \\
\hline Aragón & 2,2 & $0,04 \%$ & 901 & $0,19 \%$ \\
\hline Asturias & 18,8 & $0,47 \%$ & 6.399 & $1,56 \%$ \\
\hline Baleares & 2,9 & $0,10 \%$ & 1.006 & $0,26 \%$ \\
\hline Canarias & 3,3 & $0,05 \%$ & 2.434 & $0,35 \%$ \\
\hline Cantabria & 1,5 & $0,07 \%$ & 1.850 & $0,92 \%$ \\
\hline Castilla-La Mancha & 1,5 & $0,02 \%$ & 397 & $0,06 \%$ \\
\hline Castilla y León & 11,9 & $0,12 \%$ & 2.147 & $0,23 \%$ \\
\hline Cataluña & 71,8 & $0,29 \%$ & 12.605 & $0,48 \%$ \\
\hline Extremadura & 1,1 & $0,02 \%$ & 473 & $0,13 \%$ \\
\hline Galicia & 18,3 & $0,17 \%$ & 5.723 & $0,59 \%$ \\
\hline Madrid & 46,5 & $0,26 \%$ & 8.681 & $0,40 \%$ \\
\hline Murcia & 0,6 & $0,01 \%$ & 286 & $0,06 \%$ \\
\hline Navarra & 7,4 & $0,19 \%$ & 2.269 & $1,02 \%$ \\
\hline País Vasco & 186,6 & $2,13 \%$ & 36.004 & $4,45 \%$ \\
\hline La Rioja & 0,6 & $0,05 \%$ & 325 & $0,28 \%$ \\
\hline Comunidad Valenciana & 4,4 & $0,03 \%$ & 2.470 & $0,14 \%$ \\
\hline Ceuta & - & - & - & - \\
\hline Melilla & 0,7 & $0,35 \%$ & 263 & $1,47 \%$ \\
\hline TOTAL & 416,9 & $0,26 \%$ & 103.071 & $0,64 \%$ \\
\hline
\end{tabular}

* El dato del gasto corresponde a los presupuestos del pago de las prestaciones de renta mínima. Sin embargo, algunas Comunidades Autónomas incluyen en este dato el gasto en programas de empleo protegido o en programas de actividades dirigidos a los beneficiarios de la prestación.

** Calculado sobre el presupuesto inicial consolidado de cada Comunidad Autónoma, Ministerio de Administración Pública (<http://www.map.es>).

*** Total de hogares de Comunidades Autónomas según Encuesta Continua de Vida 2007, INE 2007 (<http://www.ine.es>).

90 Fuente: Elaboración propia a partir de datos de la Dirección General de Política Social, MEPSYD (2008). 
Asimismo, resulta obvio concluir que el incremento del gasto en estas políticas a lo largo del periodo se debe a sus incrementos solo en algunas Comunidades Autónomas. La evolución de este indicador (Gráfico 2) permite ver cómo que el País Vasco ha cuadriplicado la cifra inicial y en otros casos -andaluz, catalán, madrileño, asturiano, navarro o gallego- la cifra se ha duplicado. Sin embargo, en otro grupo de Comunidades Autónomas, las cuantías dedicadas a este programa no se han modificado o, incluso, han disminuido (casos de Cantabria, Canarias, Canarias, o Murcia).

El mismo tipo de lectura se puede hacer si atendemos a los niveles de cobertura, es decir teniendo en cuenta el número de hogares que son o han sido beneficiarios de esta medida durante el año en cuestión (Gráfico 3). El progresivo aumento de la población cubierta (63.714 en 1997 hasta 103.071 hogares en 2007, del 0,5\% al 0,6\% del total de hogares de forma aproximada) corresponde a las RMI de ciertas Comunidades Autónomas, principalmente el País Vasco (un 4,45\% de sus hogares), junto con Asturias (1,56\%) Melilla (1,47\%) y Navarra (1,03\%). Por último, otros programas de RMI, como los de Andalucía, Canarias o Galicia, apenas superan una cobertura del o,6\%.

Las tasas de coberturas han seguido a lo largo del periodo evoluciones dispares. El incremento de cobertura ha sido constante en el País Vasco, mientras en otras Comunidades Autónomas se han mantenido las tasas desde el comienzo del periodo. Tras las reformas muchas de las Comunidades Autónomas registran aumentos significativos en sus tasas de cobertura (Asturias, por ejemplo) que son seguidas por un freno en este crecimiento (Andalucía) e, incluso por llamativos descensos (Navarra o Madrid).

Dados estos niveles cobertura, y con la excepción del País Vasco, cabe evaluar que la respuesta que estos programas ofrecen a la población en situación de pobreza severa (entre el $3-4 \%$ de los hogares) es claramente insuficiente y muy inferior a la de otros mecanismos del sistema de protección social (Arriba y Guinea, 2008).

El tercer indicador explora la intensidad de la protección que ofrecen las RMI a través de las cuantías que proporcionan según el tamaño del hogar que lo percibe (Gráfico 4). No hay que olvidar que, al ser diferenciales, son las cuantías más elevadas que pueden recibir, puesto que de ellas se descuenta los ingresos que los hogares perciben por otras vías. Se trata además de las cuantías legalmente establecidas en las diferentes regulaciones autonómicas, lo que nos permiten compararlas entre sí y con otros mínimos establecidos también legalmente -Salario Mínimo Interprofesional (SMI), el Índice Público de Referencia de Efectos Múltiples (IPREM) y las Pensiones no Contributivas (PNC)-, en tanto que representaciones de lo que se considera mínimos protegibles, con los que hacer frente a las necesidades básicas.

Las cuantías básicas, que corresponden a hogares unipersonales, no muestran grandes diferencias. Se trata de cuantías muy inferiores a las que ofrece la participación laboral (SMI), comparables con las de las PNC en la mayoría de los casos o incluso inferiores (Murcia o Melilla). En el País Vasco, tras varios años de incrementos, la cuantía 


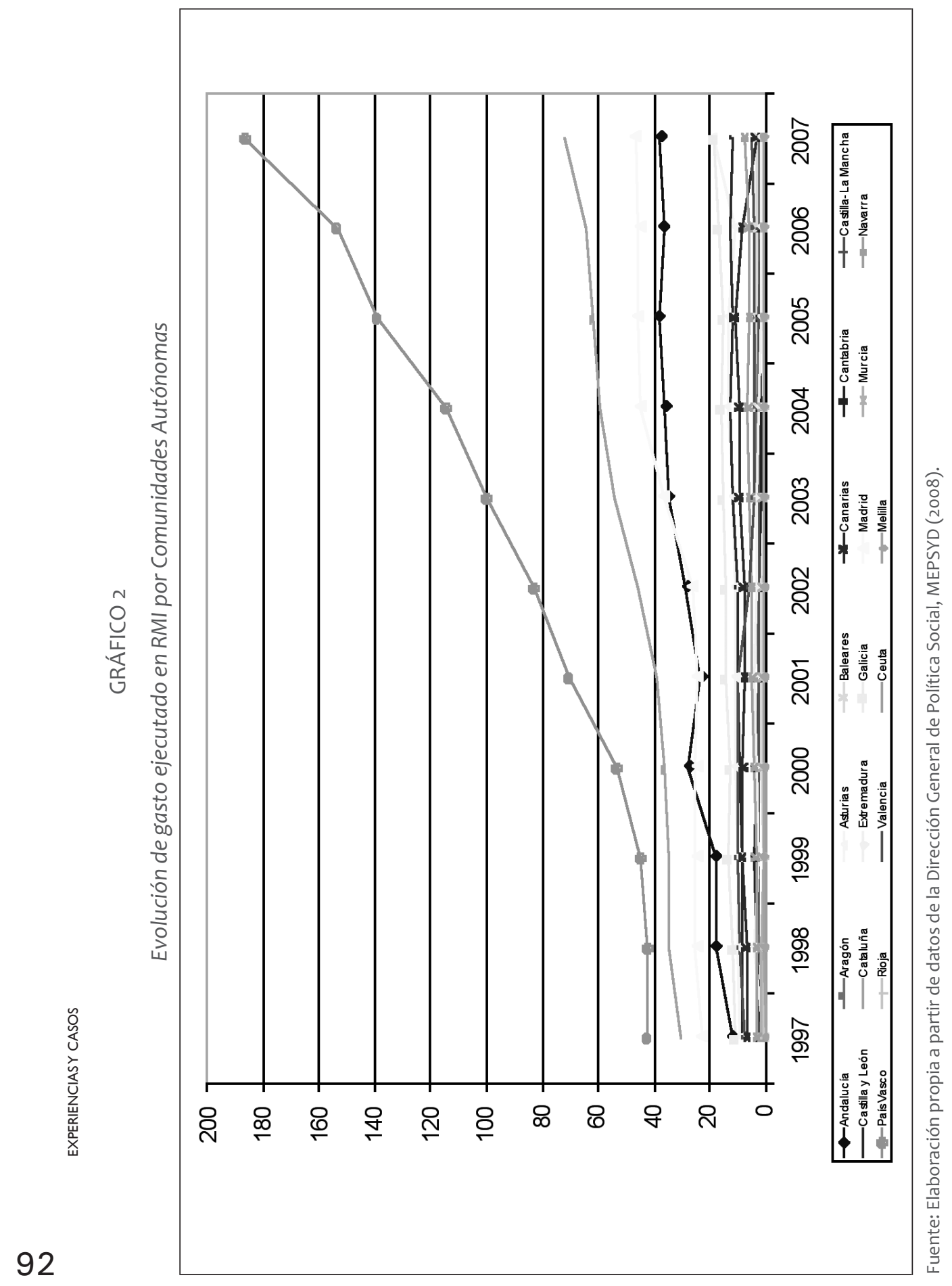


RENTAS MÍNIMAS DE INSERCIÓN DE LAS COMUNIDADES AUTÓNOMAS: UNA VISIÓN CONJUNTA DE SU EVOLUCIÓN...

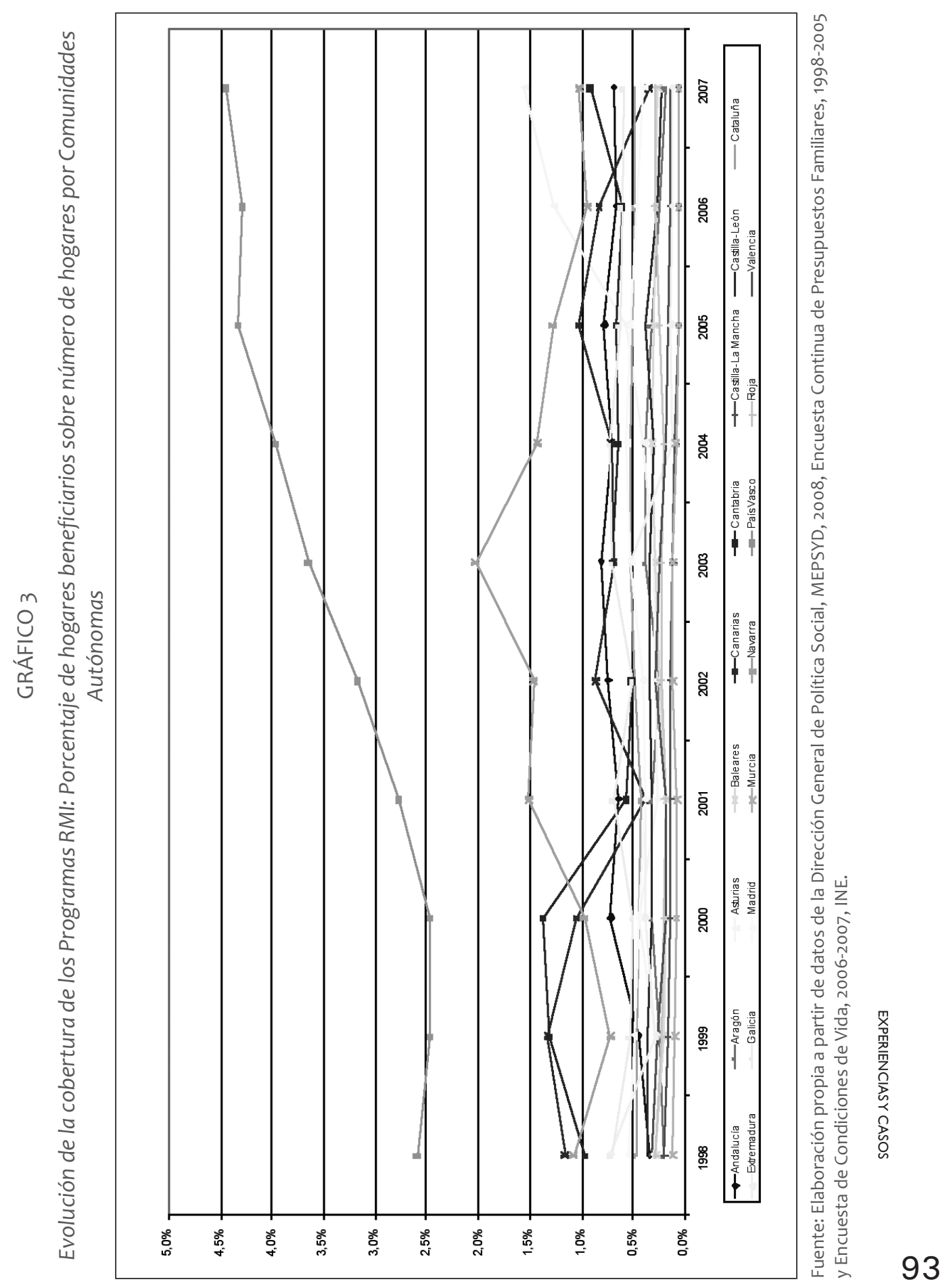

$\mathrm{G}_{\mathrm{P}}^{\mathrm{a} P}$ 


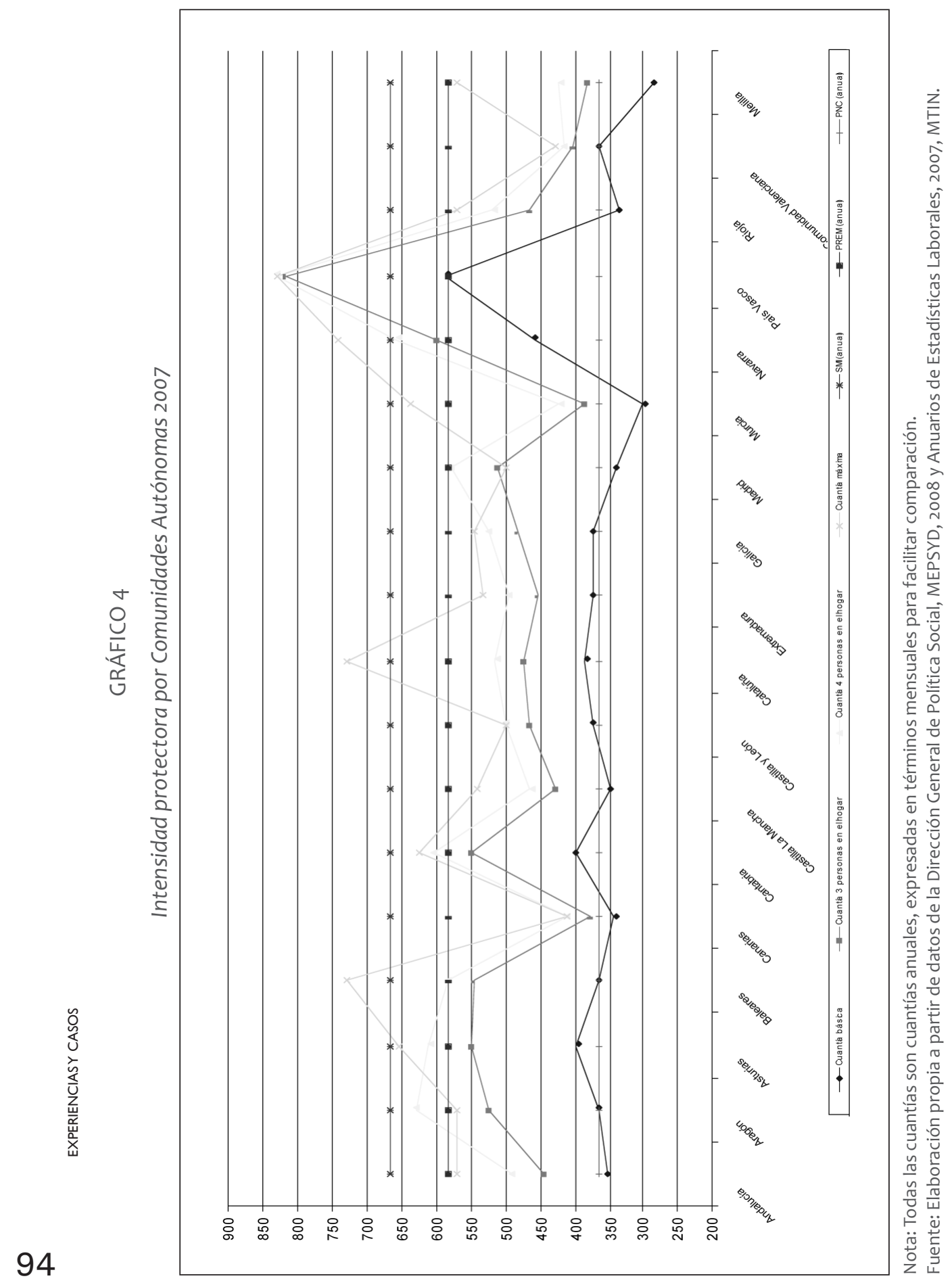


alcanza la cuantía del IPREM una referencia común para prestaciones distinto del SMI. En cualquier caso, el establecimiento de estas cuantías refleja el temor de los decisores autonómicos a que las RMI supongan un desincentivo a la participación laboral.

Sin embargo, estas cifras sobre cuantías básicas no reflejan las amplias disparidades regionales, ya que para valorar la intensidad protectora real se ha de valorar también los complementos por tamaño familiar o para cubrir necesidades específicas. Respecto a las primeras, en el Gráfico 4, se observan grandes disparidades regionales: mientras en algunas Comunidades Autónomas los complementos por tamaño del hogar (tres o cuatro personas) significan diferencias significativas en su intensidad protectora (Aragón, Baleares, Cantabria, Madrid o País Vasco) en otras se trata de una protección casi plana, no progresiva (Canarias o Comunidad Valenciana). Por último, se recogen las cuantías máximas, es decir el tope establecido en la cantidad mensual que puede recibir un unidad familiar u hogar, independientemente de su tamaño o de los ingresos que perciba por otras fuentes. En estas cuantías, la diversidad y heterogeneidad es la norma. En cuatro Comunidades Autónomas (Baleares, Cataluña, Navarra y País Vasco) se permite a la cuantía mensual superar el mínimo obtenible a través de la participación laboral, SMI. En un grupo esta cuantía se sitúa en torno al IPREM y en otro bastante amplio, se sitúa a niveles muy inferiores.

En términos generales, a pesar de que sus cuantías básicas sean similares, la intensidad protectora es muy diversa entre las Comunidades Autónomas. A esta diversidad cabría añadir otros elementos no analizados pero que en último término significan mayor disparidad protectora como son los complementos para necesidades específicas (por ejemplo vivienda) o para incentivar la participación laboral (ambos impulsados en el País Vasco).

A pesar de que no se disponen de datos sobre las formas y alcances de los complementos que las RMI ponen en marcha para alcanzar sus objetivos de inclusión social, en último término activación laboral, se recogen a continuación a las conclusiones de otros trabajos e investigaciones. En general, los estudios muestran la escasa eficacia de los mecanismos de activación con los colectivos más excluidos, algo que no es exclusivo del caso español (Arriba y Pérez-Eransus, 2007). A pesar de que estudios sobre su efecto empleando métodos econométricos muestran una ligera influencia de la participación en programas de activación (formación, orientación) (Ayala y Rodríguez, 2007), la mayoría de las evaluaciones muestran que, en realidad, favorecen la vuelta al empleo de las personas con mejores niveles de cualificación y menores niveles de exclusión social (es decir carentes de problemas adicionales como cargas familiares no compartidas, conflictividad personal, salud, discapacidad o salud mental). Sin embargo, apenas tienen efecto en la inserción laboral de aquellos que llevan más tiempo vinculados a los mecanismos de asistencia social o situación más intensas de exclusión social (Pérez-Eransus, 2005; Serrano y Arriba, 2002). Para este tipo de hogares, la opción de la vuelta al empleo no es adecuada y la continuidad en la garantía de ingresos constituye un mecanismo de elevada capacidad integradora. De hecho, la creciente introducción de condicionamientos laborales en la protección 
económica puede estar aumentando su vulnerabilidad al no poder cumplir con lo establecido en los programas de activación.

\section{CONCLUSIONES}

El desarrollo a comienzos de los años noventa de programas asistenciales como las RMI autonómicas cubrió a menor coste huecos del sistema de protección social y se orientó a atender situaciones de mayor necesidad y precariedad. Con posterioridad, el desarrollo del sistema de protección se ha centrado en la racionalización y el control de gasto, por lo que estas políticas han frenado su desarrollo. A pesar de lo cual, las RMI autonómicas han vivido también una serie de reformas y transformaciones siguiendo un itinerario desigual y heterogéneo.

Entre 1997 y 2007, mientras unas Comunidades Autónomas han emprendido procesos de reforma y mejora de sus programas de RMI, otras no les han prestado gran atención. Ni el ciclo económico ni las transformaciones socio-demográficas parecen haber influido en la diferencia de itinerarios: todas las Comunidades Autónomas han disfrutado de un periodo de crecimiento económico y bajas tasas de desempleo. La lógica de estas transformaciones sigue estando más bien en las interacciones en las propias arenas políticas. Las políticas dirigidas a la pobreza tienen para sus promotores (gobiernos autonómicos y partidos que los ocupan, partidos en la oposición, sindicatos... ) una alta capacidad de legitimación a un bajo coste. Por ello, los programas de RMI son empleados como signos de compromiso social, con escasa dedicación presupuestaria.

En este sentido podemos interpretar que los ambiciosos cambios en las normativas reguladoras, en las legislaciones, no hayan tenido una traducción cuantitativa en términos de porcentaje de gasto o que cuando lo han hecho ésta sea puntual, sin continuidad en el tiempo. La excepcionalidad del País Vasco o el hecho de que los programas que lograron mayores desarrollos en los noventa los hayan tenido también en estos procesos de reformas, se explican en buena medida por las redes de apoyo político que se establecieron en sus momentos fundacionales.

Observando la evolución anual de cobertura, intensidad o esfuerzo presupuestario por Comunidades Autónomas, resulta imposible deducir la existencia de pautas comunes de cambio. Sí se puede concluir que los itinerarios autonómicos están dando lugar a una creciente divergencia protectora: mientras algunas Comunidades Autónomas han mejorado sus regulaciones e incrementado sus niveles de gasto, cobertura e intensidad, otras los han mantenido e incluso los han disminuido. Muchas de las iniciativas de interés han tenido lugar en programas RMI que habían tenido mayores desarrollos en los periodos anteriores, en una especie de "efecto Mateo" (País Vasco, Asturias, Navarra, Madrid o Cataluña). Las diferencias a las que hacían referencia los trabajos de mediados los años noventa se mantienen, cuando no se incremen96 tan: el programa del País Vasco sigue siendo una excepción con un grado de desarro- 
Ilo aceptable; otro grupo de programas mantiene su funcionamiento como renta mínima de inserción con elementos restrictivos; por último, otro grupo ofrece una limitada y discrecional prestación asistencial que difícilmente podría denominarse renta mínima.

Las RMI son el último mecanismo de protección frente a la pobreza y la exclusión social, la "última red de seguridad”, residual y subsidiaria respecto de otros esquemas de protección. Se ha comprobado que, si bien las rentas mínimas suponen un alivio para las familias en situación de pobreza, no permiten superar el umbral de pobreza. Su bajo nivel de intensidad y cobertura protectora, además de su desigualdad territorial, les restan eficacia y dejan sin cobertura a grupos sin acceso a otros mecanismos protectores: familias con hijos, mujeres viudas o nuevas situaciones de vulnerabilidad como la de algunas poblaciones inmigrantes, trabajadores discontinuos, parados que han agotado prestaciones o no han cubierto mínimos, personas con edades cercanas a los 65 años que conviven con otros, o con minusvalías cercanas al 65\%, jóvenes sin acceso a protección... Además, el cambio que supone la orientación de las políticas asistenciales de garantía de mínimos hacia la inclusión en lugar de la pobreza ha conllevado una mudanza hacia prácticas individuales de inclusión activa basadas en la vuelta al mercado laboral. Como ya se ha indicado, esto implica desprotección para aquellas personas cuya situación de exclusión no hace viable su inserción laboral.

Parece lógico pensar que reforzar y armonizar la cobertura y la calidad de las prestaciones de RMI así como extender el reconocimiento de derechos vinculados a esta última red constituiría un importante avance en la lucha contra la pobreza y la exclusión en España. Estas medidas tendrían que ir acompañadas por dispositivos o servicios destinados a favorecer la disponibilidad para el trabajo, de intervención social de carácter integral o dispositivos de empleo de carácter ocupacional para aquellas situaciones de personas con problemas. En definitiva, plantear la inclusión como un objetivo a alcanzar mediante la sinergia de intervenciones que tienen que ver con la protección del mercado laboral, políticas de vivienda, sanidad y educación, más allá mecanismos de incentivos o condicionamientos individuales, impulsando y dotando de sentido los planes transversales o multidimensionales.

Por último, sería necesario acompañar estas medidas con mejoras en la recogida de información, armonizada y homogénea, que permitan conocer la extensión de las RMI junto con las situaciones de exclusión a las que se enfrentan. Tanto la armonización política como la informativa requieren de un compromiso activo de coordinación entre los diferentes agentes y niveles implicados en estas políticas. 


\section{REFERENCIAS BIBLIOGRÁFICAS Y DOCUMENTALES}

Aguilar, M., Gaviria, M. y Laparra, M. (1995), La caña y el pez. El salario social en las Comunidades Autónomas 1989-1994. Madrid: Fundación FOESSA.

Aguilar, M., Laparra, M. y Pérez, B., coords. (2002), La exclusión multidimensional en el espacio urbano: Investigaciones de base para la elaboración del Plan de Lucha contra la Exclusión Social en la Comunidad de Madrid. Madrid: UPN-CM.

Arriba, A. (2002), "Procesos de implantación de políticas de rentas mínimas de inserción en España", en L. Moreno ed., Pobreza y exclusión: la "malla de seguridad" en España. Madrid: CSIC.

Arriba, A. y Guinea, D. (2008), “Protección social, pobreza y exclusión social: el papel de los mecanismos de protección de rentas", en VV.AA., VI Informe sobre exclusión y desarrollo social en España 2008. Madrid: FOESSA.

Arriba, A. y Pérez-Eransus, B. (2007), “La última red de protección social en España: prestaciones asistenciales y su activación”, Política y Sociedad, 44(2): 115-133.

Aust, A. y Arriba, A. (2005), "Towards activation? Social assistance reforms and discourses” en Taylor-Gooby, P. ed., Ideas and Welfare State Reform in Western Europe. Nueva York: Palgrave-Macmillan.

Ayala, L. (2000), Las Rentas Mínimas en la reestructuración de los Estados de Bienestar. Madrid: Consejo Económico y Social.

Ayala, L. y Rodriguez, M. (2007), "What determines exit from social assistance in Spain?”, International Journal of Social Welfare, 16(2): 168-182.

Estévez, C. (1998), Las rentas mínimas autonómicas: estudio de las normativas reguladores y análisis jurídico de las prestaciones. Madrid: Consejo Económico y Social.

EAPN (2006), “Los informes estratégicos nacionales 2006-08 sobre protección social y la inclusión social. ¿Cuál es su impacto en las personas en situación de Pobreza?" En línea: <http://www.eapneuskadi.net/web/archivos/documentosyescritos/europea/Informes_Nac_2006-8.pdf> (Consulta: 21 de diciembre de 2008).

Euzeby, C. (1991), Le revenu minimum garanti. París: La Découverte.

Gobierno de Navarra (1999), Plan de Lucha contra la Exclusión en Navarra, 1998-2005. Pamplona: Gobierno de Navarra.

Laparra, M. (2004), “La travesía del desierto de las rentas mínimas en España”, Documentación Social, 135 (octubre-diciembre): 57-76.

Laparra, M., Pérez-Eransus, B., Sarasa, S., Renes, V., Pérez-Yruela, M., Trujillo, M., Subirats, J. y Obradors, A. (2007), "Una propuesta de consenso sobre el concepto de exclusión social”, Revista del Tercer Sector, 5 (enero-abril): 15-57.

Moreno, L. y Serrano, A. (2007), “Europeización del bienestar y activación”, Política y Sociedad, 44 (2): 31-44.

Pérez-Eransus, B. (2005), Políticas de activación y rentas mínimas. Madrid: FOESSA.

Pérez-Eransus, B., Arriba, A. y Parrilla, J.M. (2009), "Transformaciones de las políticas autonómicas de inclusión social: ¿Reforma o cambio de imagen?” en L. Moreno. ed., Reformas de las políticas de bienestar en España. Madrid: Siglo XXI.

98 clusión Social en el Principado de Asturias. Madrid: CSIC. 
Pérez-Yruela, M., Sáez, H. y Trujillo, M. (2002), Pobreza y exclusión social en Andalucía. Madrid: CSIC.

Rodriguez Cabrero, G. (2004), El Estado del bienestar en España: debates, desarrollo y retos. Madrid: Fundamentos.

Taylor-Gooby, P. (2004), New Risks, New Welfare: The Transformation of the European Welfare State. Oxford: Oxford University Press.

Serrano, A. y Arriba, A. (2002), “El Ingreso Madrileño de Integración: Revisitando sus características y algunos tópicos", en L. Moreno, ed., Pobreza y exclusión: la "malla de seguridad" en España. Madrid: CSIC.

Serrano, A. y Arriba, A. (1998), ¿Pobres o excluidos? El Programa del Ingreso Madrileño de Integración en perspectiva comparada. Madrid: Fundación Argentaria-Ministerio de Trabajo y Asuntos Sociales-Editorial Visor.

VV.AA. (2008), VI Informe sobre exclusión y desarrollo social en España 2008. Madrid: FOESSA. 